\title{
Improving Operator Performance through the Use of a Multivariable Human-Machine Control Strategy
}

\author{
Rui Antunes ${ }^{1,3}$, Fernando V. Coito ${ }^{1,2}$, and Hermínio Duarte-Ramos ${ }^{2}$ \\ ${ }^{1}$ UNINOVA \\ ${ }^{2}$ Departamento de Engenharia Electrotécnica, Faculdade de Ciências e Tecnologia da \\ Universidade Nova de Lisboa \\ 2829-516 Caparica, Portugal \\ \{fjvc, hdr\} @eft.unl.pt \\ ${ }^{3}$ Escola Superior de Tecnologia de Setúbal do Instituto Politécnico de Setúbal \\ 2910-761 Estefanilha, Setúbal, Portugal \\ rui.antunes@estsetubal.ips.pt
}

\begin{abstract}
Most of the mechatronics equipments and gadgets that we all nowadays rely on incorporate some kind of multidimensional human-machine systems. There is an increasing concern for improving the usability, performance, ergonomics and safety of such devices, and ultimately this will lead to the mass-production of next-generation intelligent machines, which will be capable to assist the human operator and to reduce the global effort by estimating and measuring its skills. This ongoing work introduces a novel human-machine multi-dynamic modeling methodology which can be applied on the development of these Human Adaptive Mechatronic (HAM) machines, able to adapt to the skill/dexterity levels of its users, and to enhance Human's proficiency. As a new strategy for model development, a number of twodimensional independent pursuit manual tracking experiments are evaluated. A human-machine state-space linear model is obtained and successfully applied to design an improved closed-loop multivariable control structure.
\end{abstract}

Keywords: Human-Machine Dynamics, Man-Machine Interfaces, Human-inthe-Loop Multivariable Control, Manual Tracking Systems.

\section{Introduction}

Today, the design and development of intelligent human-machine devices and assisted-control schemes is becoming a growing field of research. This trend is due to the fact that the overall performance in any human-machine process, in terms of energy cost, productivity, quality and safety depends both on the machine technical condition and on the skills of human user. Recent studies [1] revealed that in productive processes involving manual operations (such as in forestry, construction, agriculture and mineral plants), the human operator impact factor in the overall performance can reach over $40 \%$. Also, in many other research areas, as in medicine, biotechnology, space, transports, entertainment, nanotechnology and ocean, where the need for assisted machines is crucial, there is nowadays a strong demand to increase 
safety, accuracy and precision. To meet this goals new intelligent assisted-control strategies [2], [3], [4] where introduced which intend to estimate the human operator behavior, and produce an assist-force according to the estimated skill level.

Human-Machine dynamics depends on the human factor, the machine condition, and also on the type of manual tasks involved (often taking place in more than a single spatial dimension). Hence, whatever modeling and control strategy should be taken to improve performance, it is obvious that we are generally dealing with complex multiple-input and multiple-output (MIMO) system architectures.

\section{Contribution to Value Creation}

The aim of this work is to present a contribution on human-machine dynamic modeling for control purposes taking place on 2-D environments. The inclusion of these models in a closed-loop multivariable control scheme makes the human operator to take less time and effort to become skillful, and leads also to an improved performance on 2-D manually controlled tasks. The research results from this work serve as a basis for developing a human adaptive mechatronics multivariable control framework, which can be applied on many applications ranging from human operated manufacturing environments, tele-robotics, robot-assisted surgery, space and marine environments, automotive industry, aviation, etc.
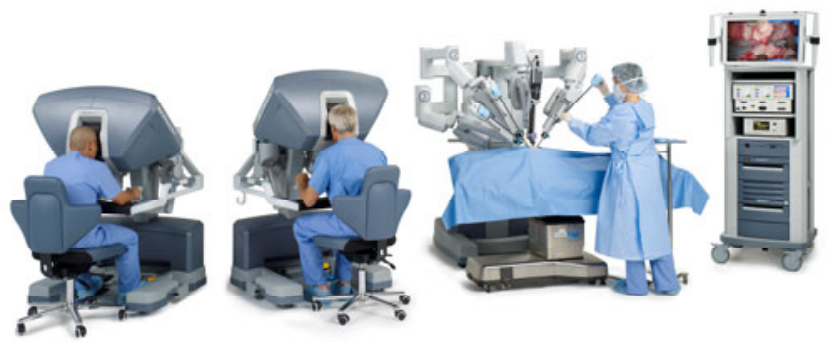

Fig. 1. Robot-assisted laparoscopic surgery (@2011 Intuitive Surgical, Inc.)

The impact of human performance on world growth, on the environment protection, health care and comfort can be viewed as a direct consequence of previous investments made in technological innovation for value creation. In addition there is nowadays a strong interest in developing new evaluation metrics, data monitoring and experimental procedures required for quantifying the human-machine interaction skills, which are also proposed and described with detail in this paper.

\section{State-of-the-Art / Related Literature}

It is clear that if we want to design a human-oriented machine we need to model the human operator characteristics. Unfortunately, the human element is the most 
complex component to study in a human-machine device. The operator behavior may alter according to many intrinsic factors, such as concentration, physical condition, fatigue or training, and also with other external causes (disturbances, comfort, etc.), including the type of manual task being performed. The fact that human behavior always results from a set of feedback loops only stresses the difficulty on modeling the operator.

The Human Adaptive Mechatronics (HAM) concept [2] was first launched between 2003 and 2008 at Japan, in Tokyo Denki University, as result of a scientific research project aimed to intelligently assist the human operator in improving its skills. A HAM assist control system [2] uses an intelligent control scheme which identifies operator individual characteristics based on its manual actions. An adaptive controller then provides an assist force for the operator, to improve task performance on the machine, according to previous measured/estimated skill.

Investigation on HAM has spread to other countries and is nowadays a field of research supported by many Universities, I\&D Centers and Companies. Recent advances on HAM research include the development of mobile working machines, adaptive assistance for mobile vehicles, teleoperation and coaching systems [5], [6]. Other promising research areas are assisted laparoscopic surgery [7], and humanmachine coordination using Hidden Markov Models for recognizing actions in haptic devices and virtual environments [8]. Cutting-edge research for obtaining the human operator dynamics covers neuro-fuzzy and fuzzy-ARX modeling techniques [9], the modified optimal control model (MOCM) method and particle swarm optimization [1]. Other areas of research also emphasis the problem of skill quantification [10] and the Fitts speed-accuracy trade off [11] during a manual task.

\section{$4 \quad$ Research Contribution and Innovation}

Operator task response generally does not follow a linear behavior due to the human's non-linear time-varying systemic complexity. However, it was been shown that linear models can still be used as a way to capture some of the relevant characteristics of human-machine systems, specially in simple servo/regulator manual control tasks [12]. Previous 1-D tracking experiments have also revealed that the human dynamics cannot be fully described with a single LTI model [13]. Moreover, in any manual task the operator closes the loop between sensing and acting, which makes harder to obtain the open-loop models involved.

\subsection{The Human-Machine Modeling Strategy}

State-space linear system representations can be obtained directly from physical (mathematical) modeling (which may be a complex and time-consuming task), or through various system identification methods. The scope of the present work is focused on this second option, which addresses the multivariable system identification problem. i.e. on how to obtain a human-machine MIMO model from the multiinput/output closed-loop collected data. 
As a first approach for modeling in 2-D environments, we simplify the problem by assuming that a human-machine interface may be estimated as a linear system which is confined on a two-independent orthogonal axes scheme $(\mathrm{X}, \mathrm{Y})$, where the influence on the output response in one axis is independent from the other axis. By adopting this approach, the human-machine MIMO system may be modeled from two independent linear transfer functions, leading to a diagonal state-space dynamic matrix pattern. Each axis input-output transfer function can then be experimentally obtained from several pursuit manual tracking experiments, using the frequency analysis methods already developed in previous work [13], [14], [15].

The frequency analysis method was used to obtain each of the axis input-output transfer functions, assuming a human-machine linear time-invariant (LTI) approximation. According to the procedure described in [13] for SISO modeling, a reference (target) signal $x(t)$ is produced for the human operator to track, and $y(t)$ is the equivalent linear human response. The target signal $x(t)$ is built from a set of certain multiple frequencies, chosen in order to cover the manual operation frequency spectrum. This procedure ensures that only the present frequencies in the reference signal are taking into account for modeling purposes. The reference signal $x(t)$ is generated in a certain way to avoid any "target learning" effect. The obtained model contains the closed-loop behavior of the human operator:

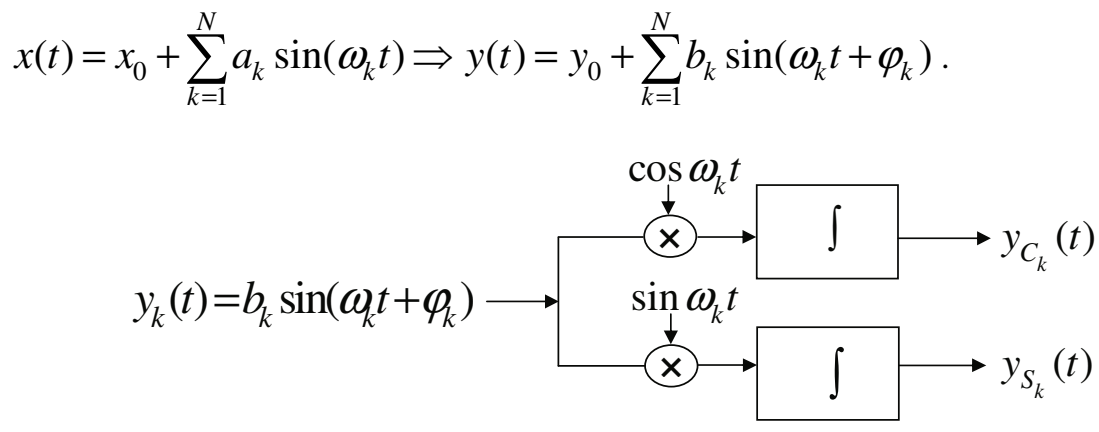

Fig. 2. Frequency analysis block diagram for each $k$-multiple frequency

By integrating along time $T=\frac{k 2 \pi}{\omega}$ (as a multiple of the sinusoid period), leads to:

$$
\begin{aligned}
y_{C_{k}}(T) & =\int_{0}^{T} b_{k} \sin \left(\omega_{k} t+\varphi_{k}\right) \cos \omega_{k} t d t \Leftrightarrow y_{C_{k}}(T)=\frac{b_{k} T}{2} \sin \varphi_{k} . \\
y_{S_{k}}(T) & =\int_{0}^{T} b_{k} \sin \left(\omega_{k} t+\varphi_{k}\right) \sin \omega_{k} t d t \Leftrightarrow y_{S_{k}}(T)=\frac{b_{k} T}{2} \cos \varphi_{k} .
\end{aligned}
$$

$$
b_{k}=\frac{2}{T} \sqrt{y_{C_{k}}{ }^{2}(T)+y_{S_{k}}{ }^{2}(T)} \text { and } \varphi_{k}=\arctan \left(\frac{y_{C_{k}}(T)}{y_{S_{k}}(T)}\right) \quad K_{0}=\frac{y_{0}}{x_{0}} \text {. }
$$

Which corresponds, for each independent axis, to the closed-loop human-machine frequency response and static gain $K_{0}$ (for a previous input offset $x_{0}$ ). 
By adopting this procedure, and from the experimental collected data which reflects the closed-loop human-machine behavior, two open-loop transfer functions ( $M_{x x}(s)$ and $M_{y y}(s)$ ) can be obtained for each independent axis, through inverse manipulation, and a correspondent human-machine transfer matrix representation, despising at this phase the cross-terms, can be written as:

$$
\left[\begin{array}{l}
P_{x}(s) \\
P_{y}(s)
\end{array}\right]=\left[\begin{array}{cc}
M_{x x}(s) & 0 \\
0 & M_{y y}(s)
\end{array}\right] \cdot\left[\begin{array}{l}
J_{x}(s) \\
J_{y}(s)
\end{array}\right] .
$$

Where $P_{x}(s)$ and $P_{y}(s)$ are the output responses and $J_{x}(s)$ and $J_{y}(s)$ the input targets (respectively, for the $\mathrm{X}$-axis and $\mathrm{Y}$-axis). The open-loop transfer functions $M_{x x}(s)$ and $M_{y y}(s)$ were obtained from two independent sets of pursuit manual tracking experiences, each performed for each axis. The magnitude characteristic of the input signal used is presented in figure 3 :

Input target signal for $X$-axis and $Y$-axis:

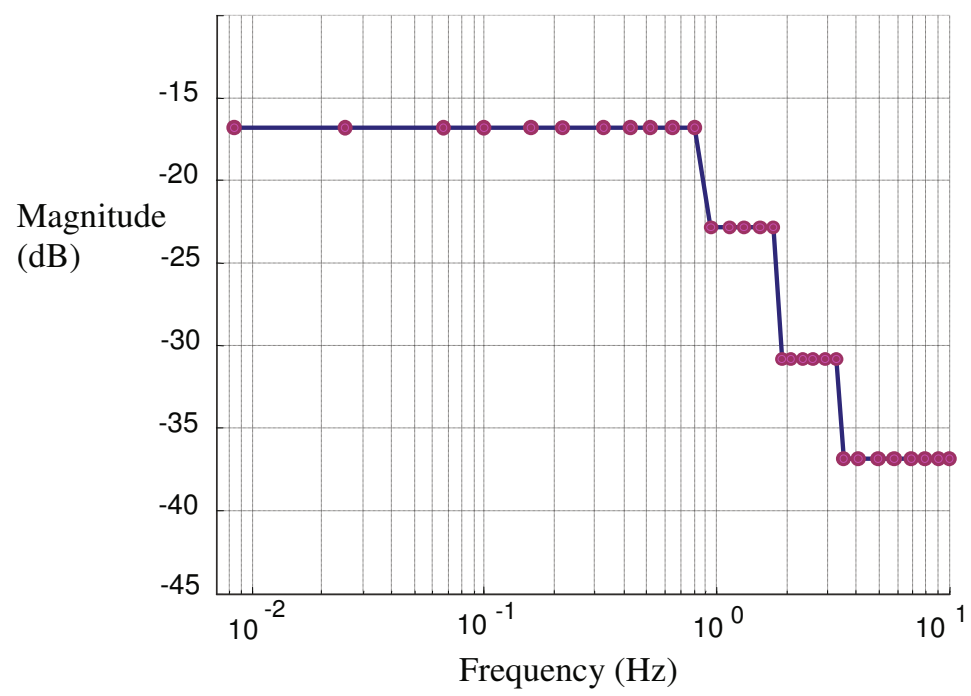

Fig. 3. Input (target) signal magnitude for the $X$-axis and $Y$-axis manual tracking experiments, based on the $\mathrm{N}=30$ frequencies sum, ranging from $0.0083 \mathrm{~Hz}$ to $10 \mathrm{~Hz}$

\subsection{2-D Pursuit Manuel Tracking}

Fifty pursuit manual tracking time-trials, each with a 120 seconds duration $(T)$, were alternatively performed for each axis, by a same participant with no history of neurological disease. A minimum 5 minute rest between trials ensured that human fatigue or memorization did not influenced results: 

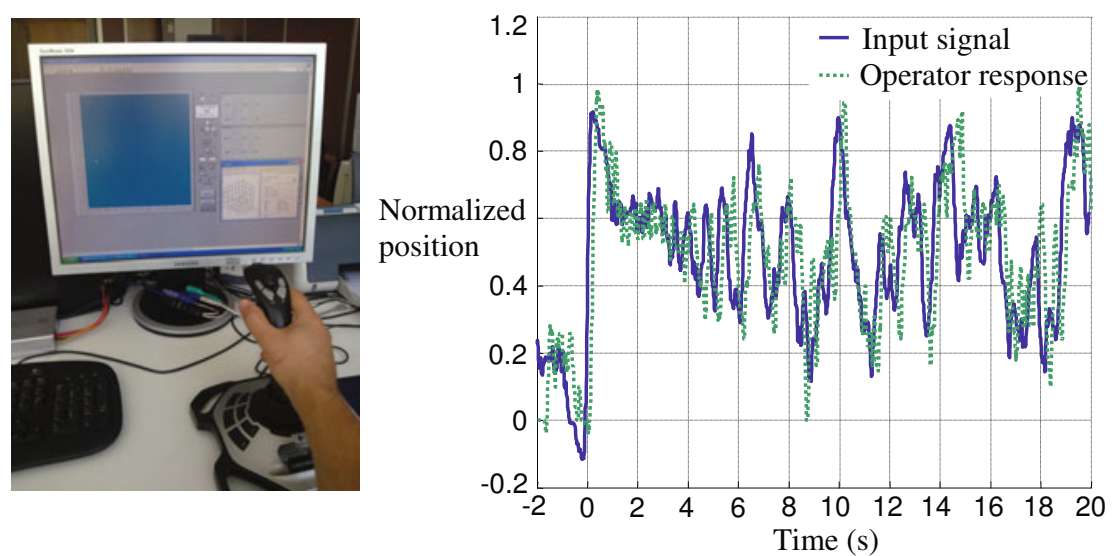

Fig. 4. A pursuit manual tracking time-trial using Logitech's Extreme 3D Pro. 8-bit analog Joystick (left). $Y$-axis tracking sample (first 20 seconds) at $100 \mathrm{~Hz}$ sampling rate (right). The input offset signal $x_{0}$ is 0.4 at 0 and at 120 seconds $(t=0, T)$.

From the collected data, two amplitude independent open-loop nominal models were obtained, one for each axis, through inverse manipulation:
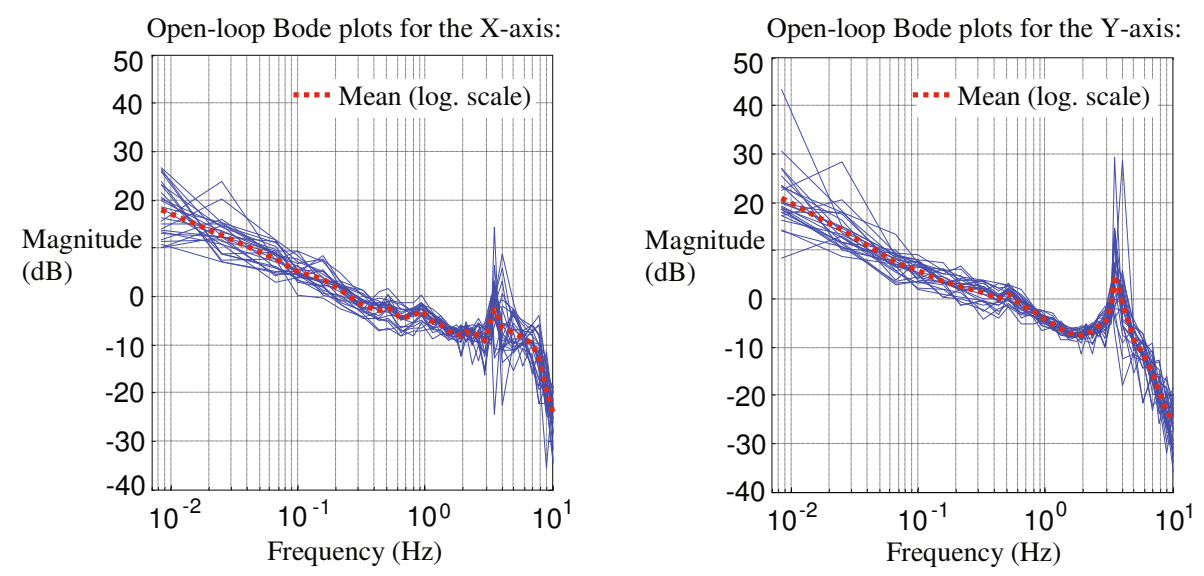

Fig. 5. Twenty-five open-loop magnitude Bode plots, for X-axis (left) and $Y$-axis (right)

Magnitude behavior is assumed similar for both the axes, and an unique 3 stable pole simplified open-loop model, for $M_{x x}(s)$ and $M_{y y}(s)$, is proposed:

$$
M_{x x}(s) \approx M_{y y}(s)=\frac{2060}{s^{3}+4.5 s^{2}+527 s+679} .
$$


The experimental procedures already described were independently performed for each axis. Therefore it should be stressed that, at this stage, the correspondent statespace simplified model will not reflect any axis cross-dependency effect.

\subsection{Multivariable Controller Design Strategy}

The human-machine interface setup was applied to an unstable MIMO process, which consisted of two equally independent double-integrators (one $P(s)$ for each axis):

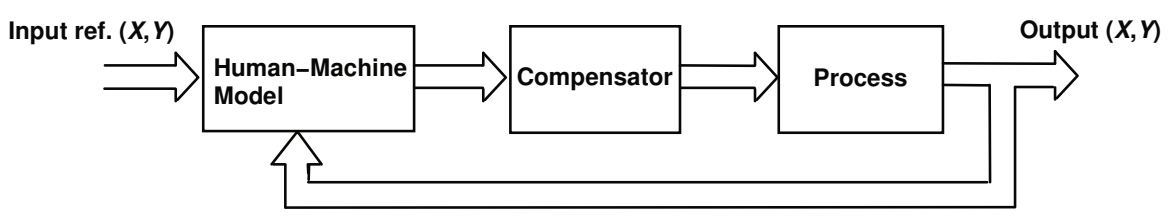

Fig. 6. Block diagram of the system to be controlled (ex: propelling a mass through space)

Two types of controllers were proposed to control the unstable system (in both $\mathrm{X}$ and $\mathrm{Y}$-axis). The first type $(C)$ is a classical lead-compensator, and the second type ( $C_{H}$ ) is obtained form $C$, taking also into account the human-machine model:

$$
\begin{gathered}
P(s)=\frac{10}{s^{2}} \quad C(s)=\frac{0.5 s+0.05}{s+50} \quad C_{H_{x x}}(s)=C_{H_{y y}}(s) \\
C_{H y y}(s)=\frac{C(s)}{F_{1}(s)}=\frac{256000 s^{4}+1.178 e 006 s^{3}+1.35 e 008 s^{2}+1.873 e 008 s+1.738 e 007}{2060 s^{4}+597400 s^{3}+6.427 e 007 s^{2}+3.032 e 009 s+5.274 e 010}
\end{gathered}
$$

Where $F_{1}(s)$ presents the same frequency behavior as (10), but includes an additional term $^{1}$ to allow the implementation of $C_{H_{x x}}(s)$ (and $\left.C_{H_{y y}}(s)\right)$.

A closed-loop state-space system representation can then be written in the form:

$$
\begin{aligned}
& \frac{d}{d t}\left[\begin{array}{l}
X_{1} \\
X_{2}
\end{array}\right]=\left[\begin{array}{cc}
A & {[0]} \\
{[0]} & A
\end{array}\right] \cdot\left[\begin{array}{c}
X_{1} \\
X_{2}
\end{array}\right]+\left[\begin{array}{cc}
B & {[0]} \\
{[0]} & B
\end{array}\right] \cdot\left[\begin{array}{l}
u_{x} \\
u_{y}
\end{array}\right] \\
& y=\left[\begin{array}{cc}
C & {[0]} \\
{[0]} & C
\end{array}\right] \cdot\left[\begin{array}{l}
X_{1} \\
X_{2}
\end{array}\right]+\left[\begin{array}{ll}
d & 0 \\
0 & d
\end{array}\right] \cdot\left[\begin{array}{l}
u_{x} \\
u_{y}
\end{array}\right]
\end{aligned}
$$

\section{$5 \quad$ Results and Critical View}

A real-time manual tracking application was developed using LabVIEW, for evaluating the operator performance at 2-D environments:

\footnotetext{
${ }^{1}$ Note that directly dividing $C(s)$ by $M_{y y}(s)$ leads to an improper transfer function (with more zeros than poles), which is not physically realizable. Thus, the need for the additional term, made from 3 non-dominant poles at $12.7324 \mathrm{~Hz}$ ( $80 \mathrm{rad} / \mathrm{s}$ ), and with unity static gain.
} 

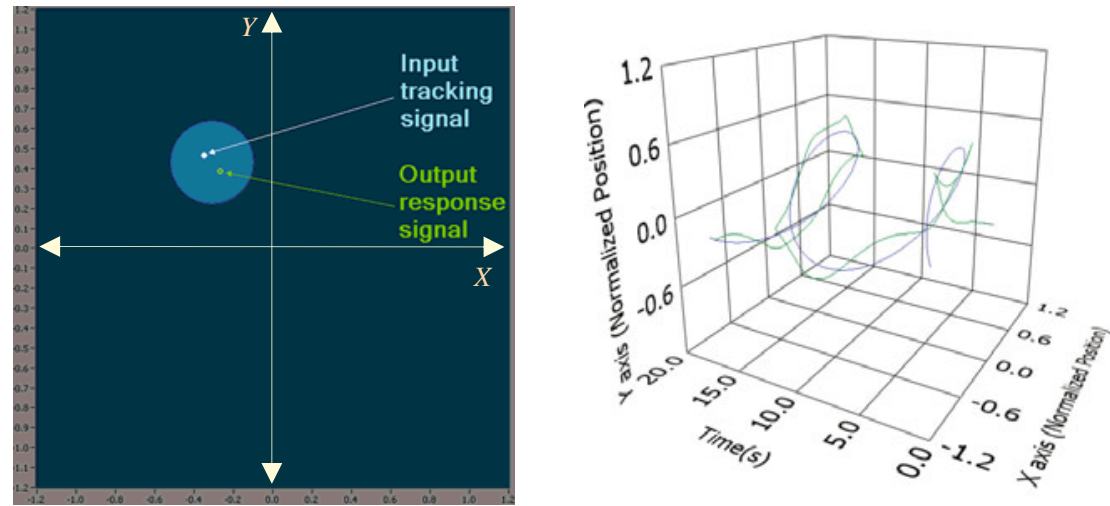

Fig. 7. LabVIEW developed application for manual tracking in an unstable process, using an analog Joystick (left). Ex: manually tracking an ellipsoid input (blue) target signal (right).

$\mathrm{MQE}=0.1815 \mathrm{MQD}=0.2051 \quad \mathrm{TMD}=1.8800 \quad 6001$ samples $100 \mathrm{~Hz}$
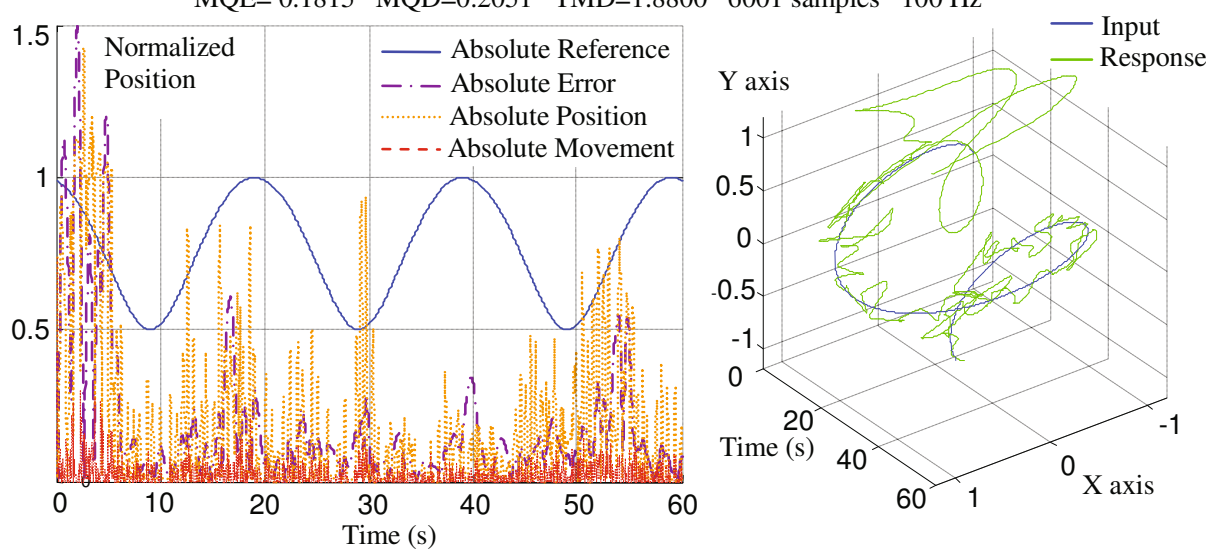

Fig. 8. Manual effort and acuity for an ellipsoidal target reference, without any compensator

\subsection{Human Skill Evaluation}

Three performance metrics were proposed, for quantifying the operator's skill in the unstable process: the mean quadratic Cartesian error (MQE) between input reference and output response, for acuity; the mean quadratic Cartesian joystick deformation (MQD - related with the amount of force applied); and finally, the total absolute value of the Cartesian movement (TMD), divided by the experiment duration (related with overall hand movement). The experimental results, shown in figures 8 and 9 , used an origin centered ellipsoid moving target (for 60 seconds), with an eccentricity of 0.866 : 

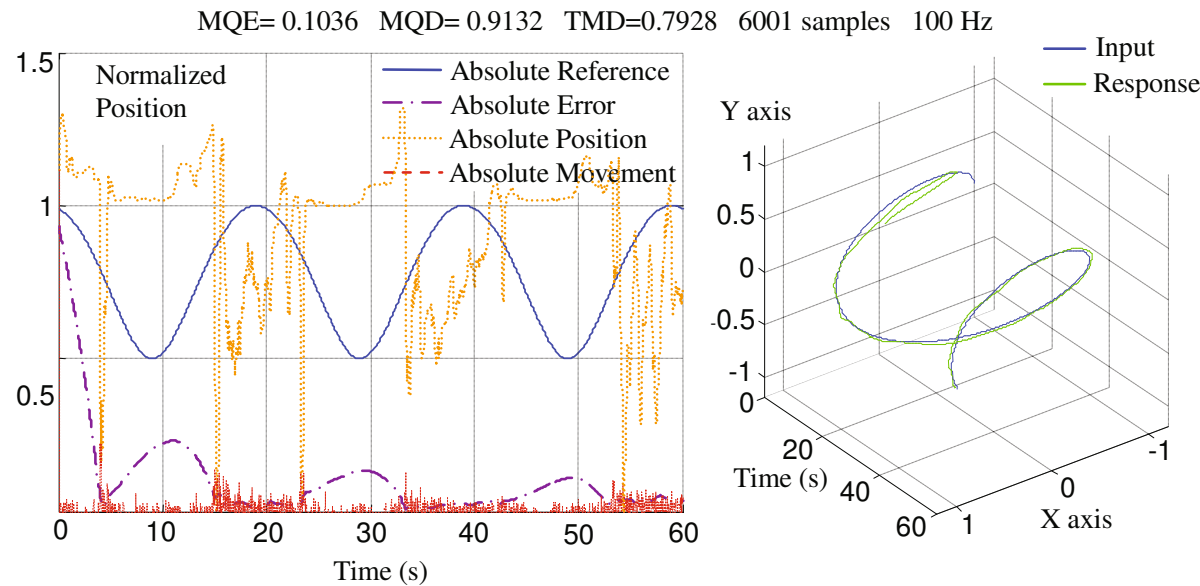

$\mathrm{MQE}=0.0412 \mathrm{MQD}=0.0175 \mathrm{TMD}=0.1541 \quad 6001$ samples $100 \mathrm{~Hz}$
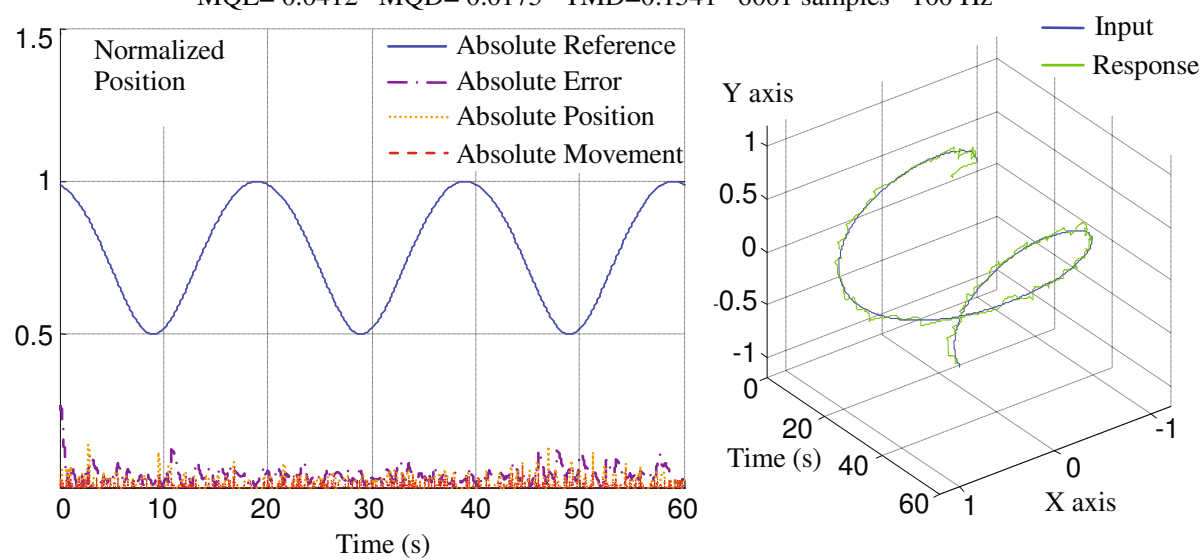

Fig. 9. Manual effort and acuity for an ellipsoidal target reference, with the lead $(C)$ compensators (up), and with the $C_{H}$ controllers (down)

The obtained experimental results show a significantly improvement in task performance, manual effort, and stability when using the proposed human-machine control scheme (with $C_{H}(s)$ type controllers) in the process dynamics, meaning also that the operator will take less time and effort to become skillful.

\section{Conclusions and Further Work}

This work introduces a multivariable human-machine modeling and control approach for enhancing human skill in bidirectional manual tasks. A 2-D human-machine interface experimental setup was built, to demonstrate the effectiveness of the proposed modeling and control methodologies. Obtained results confirmed the importance of the developed HAM strategy in improving operator performance, which is becoming particular relevant in high precision mechatronic applications. 
A future work direction lies in obtaining better human-machine realistic models that also cover the physical cross-input-output axes dependencies, combined with new skill-based HAM adaptation mechanisms, obtained, for example, from an adaptive switching robust controller or through swarm intelligence algorithms.

\section{References}

1. Tervo, K., Koivo, H.: Towards human skill adaptive manual control. Int. J. Advanced Mechatronic Systems 2(1/2), 46-58 (2010)

2. Harashima, F., Suzuki, S.: Human Adaptive Mechatronics - Interaction and Intelligence. In: 9th IEEE International Workshop on Advanced Motion Control, Istanbul, pp. 1-8 (2006)

3. Igarashi, H.: Human adaptive assist planning without operator awareness. In: IEEE Intern. Conference on Systems, Man and Cybernetics, Montreal, pp. 2933-2938 (2007)

4. Suzuki, S., Harashima, F.: Assist Control and its Tuning Method for Haptic System. In: 9th IEEE International Workshop on Advanced Motion Control, Istanbul, pp. 374-379 (2006)

5. Tervo, K., Bocca, M., Eriksson, L.M., Manninen, A.: Wireless manual control for human adaptive mechatronics. Int. J. Advanced Mechatronic Systems 2(4), 254-270 (2010)

6. Palmroth, L., Tervo, K., Putkonen, A.: Intelligent coaching of mobile working machine operators. In: Proceedings of the IEEE 13th International Conference on Intelligent Engineering Systems, Barbados, pp. 149-154 (2009)

7. Sadahiro, T., Hamazaki, M., Miyawaki, F., Yoshimitsu, K., Masamune, K.: Laparoscopic skill measurement with COP to realize a HAM Scrub Nurse Robot system. In: Proceedings of the IEEE International Conference on Systems, Man and Cybernetics, Montreal, pp. 2983-2988 (2007)

8. Palmroth, L., Putkonen, A.: Work cycle recognition in human operated machines using hidden Markov models. In: Proceedings of the 8th International Conference on Motion and Vibration Control, Daejeon, pp. 459-464 (2006)

9. Celik, O., Ertugrul, S.: Predictive human operator model to be utilized as a controller using linear, neuro-fuzzy and fuzzy-ARX modeling techniques. In: Engineering Applications of Artificial Intelligence, vol. 23, pp. 595-603. Elsevier (2010)

10. Suzuki, S., Kobayashi, H., Harashima, F.: Brain monitoring analysis of skill on voluntary motion. In: Inter. Conf. on Control, Automation and Systems, Seoul, pp. 1178-1182 (2007)

11. MacKenzie, I., Buxton, W.: Extending Fitts' law to two-dimensional tasks. In: Proceedings of the ACM Conference on Human Factors in Computing Systems, New York, pp. 219$226(1992)$

12. Gaines, B.: Linear and Nonlinear Models of the Human Controller. International Journal of Man-Machine Studies 1, 333-360 (1969)

13. Antunes, R., Coito, F., Duarte-Ramos, H.: Human-Machine Control Model Approach to Enhance Operator Skills. In: Proceedings of IEEE International Conference on Mechanical and Electrical Technology, Singapore, pp. 403-407 (2010)

14. Antunes, R., Coito, F.V., Duarte-Ramos, H.: Using Human Dynamics to Improve Operator Performance. In: Camarinha-Matos, L.M., Pereira, P., Ribeiro, L. (eds.) DoCEIS 2010. IFIP AICT, vol. 314, pp. 393-400. Springer, Heidelberg (2010)

15. Antunes, R., Coito, F.V., Duarte-Ramos, H.: A Linear Approach towards Modeling Human Behavior. In: Camarinha-Matos, L.M. (ed.) Technological Innovation for Sustainability. IFIP AICT, vol. 349, pp. 305-314. Springer, Heidelberg (2011) 